\title{
Comparison of neural activity related to working memory in primate dorsolateral prefrontal and posterior parietal cortex
}

\author{
Xue-Lian Qi, Fumi Katsuki, Travis Meyer, Justin B. Rawley, Xin Zhou, Kristy L. Douglas and \\ Christos Constantinidis*
}

Department of Neurobiology and Anatomy, Wake Forest University School of Medicine, Winston-Salem, NC, USA

\section{Edited by:}

Steven S. Hsiao, Johns Hopkins

University, USA

\section{Reviewed by:}

Veit Stuphorn, Johns Hopkins

University, USA

Mathew Chafee, University of

Minnesota, USA

${ }^{*}$ Correspondence:

Christos Constantinidis, Department of Neurobiology and Anatomy, Wake

Forest University School of Medicine, Winston-Salem, NC 27157-1010, USA

e-mail:cconstan@wfubmc.edu
Neurons in a distributed network of cortical and subcortical areas continue to discharge after the presentation and disappearance of stimuli, providing a neural correlate for working memory. While it is thought that the prefrontal cortex plays a central role in this network, the relative contributions of other brain areas are not as well understood. In order to compare the contributions of the dorsolateral prefrontal and posterior parietal cortex, we recorded neurophysiological activity in monkeys trained to perform two different visuo-spatial working memory tasks: a Match/Nonmatch task, and a Spatial Delayed-Match-to-SampleTask. Neurons in both areas exhibited discharges in the delay periods of the tasks that could be classified in two forms. Sustained discharges persisted after the presentation of a stimulus in the receptive field with a constant or declining rate. Anticipatory responses increased in rate during the delay period, often appearing after presentation of a stimulus out of the receptive field. Despite similarities, we uncovered distinct differences between patterns of delay period in each brain area. Only in the prefrontal cortex sustained responses related to the original stimulus survived presentation of a second stimulus, in the context of the Match/Nonmatch task. Our results provide insights on the nature of processing in two areas active during working memory, and on the unique role of the prefrontal cortex in memory maintenance.

Keywords: monkey, neurophysiology, vision, principal sulcus, intraparietal sulcus, persistent activity, spatial memory

\section{INTRODUCTION}

Working memory refers to the ability to maintain and manipulate information in memory over a time interval of seconds (Baddeley, 1992). Neurophysiological recordings in animal models have provided a neural correlate of working memory in the form of neuronal discharges that are elicited by physical stimuli but which persist even after the stimuli are no longer present (Fuster and Alexander, 1971; Funahashi et al., 1989). Although first described in the prefrontal cortex, persistent discharges have now been reported in most areas of the association cortex and several subcortical areas (Constantinidis and Procyk, 2004; Pasternak and Greenlee, 2005). Nonetheless, the magnitude and time course of persistent discharges differs between cortical areas, suggesting that different areas play distinct roles in the maintenance of working memory.

Early studies comparing patterns of neuronal activity in the prefrontal cortex and inferior temporal cortex revealed unique properties of the prefrontal cortex. In animals tested with a Delayed Match-to-Sample-Task, activity of prefrontal neurons activated by the sample stimulus continued to persist and survived the presentation of nonmatch stimuli that the monkeys had to ignore in order to perform the task (Miller et al., 1996). In contrast, inferior temporal neurons were activated by each stimulus appearing in sequence. Therefore their activity could only represent the most recent stimulus, whether it was the sample that needed to be remembered or a nonmatch stimulus that was to be ignored (Miller et al., 1993). Two other studies examining responses of neurons during spatial working memory reached equivalent conclusions for the prefrontal and posterior parietal cortex: Activity of Prefrontal neurons appeared to survive distracting stimulation (di Pellegrino and Wise, 1993) whereas, activity of posterior parietal neurons only tracked the most recent stimulus (Constantinidis and Steinmetz, 1996). Based on these results, it was concluded that prefrontal cortex was unique in its ability to maintain information in memory in the face of distraction. However, these studies relied on quite different behavioral tasks and it is not known to what extent the choice of task affected the pattern of responses. The only direct comparison of prefrontal and posterior parietal neurons in the same animals performing the same task relied on an oculomotor delayed response task, which did not involve a distractor, and revealed little difference between prefrontal and parietal areas in terms of delay period activity (Chafee and Goldman-Rakic, 1998).

In the last few years, some studies have challenged the idea of an absolute dichotomy between the properties of the prefrontal cortex and its cortical afferents. Neurons in inferior temporal cortex were shown to represent a stimulus retrieved from long-term memory even when distracting stimuli intervened (Takeda et al., 2005). Another recent study in the inferior temporal cortex suggested that a few inferior temporal neurons continue to represent the sample even after the presentation of a nonmatch stimulus (Woloszyn and Sheinberg, 2009). These results suggest that the properties of prefrontal cortex may be quantitatively rather than qualitatively different (Sigala, 2009). In order to determine whether this was the case for spatial working memory as well, we tested neuronal responses from the prefrontal and posterior parietal cortex of the same animals recorded during the working memory intervals of 
spatial memory tasks. Our study addresses how patterns of activity relating to spatial working memory differ between cortical areas and in the context of different tasks.

\section{MATERIALS AND METHODS}

Four male, rhesus monkeys (Macaca mulatta) weighing $5-12 \mathrm{~kg}$ were used in these experiments. All animal experiments were performed in compliance with the guidelines set forth by the National Institutes of Health as reviewed and approved by the Wake Forest University Animal Care and Use Committee.

\section{ANATOMICAL LOCALIZATION}

Neural recordings were performed in two cortical regions, the dorsolateral prefrontal cortex and posterior parietal cortex (Figure 1). Prefrontal recordings targeted areas 46 and 8 including the caudal part of both banks of the Principal Sulcus, the area between the Principal and the Arcuate sulci, and part of the superior convexity of the lateral prefrontal cortex. Posterior parietal recordings were conducted in area $7 \mathrm{a}$, an area directly interconnected with the prefrontal areas targeted in this study (Cavada and GoldmanRakic, 1989). During experimental sessions, the depths of the cortex encountered by electrodes provided a coarse map of anatomical location. Upon completion of the experiments, the anatomical location of electrode penetration was superimposed onto an image of the cortical surface obtained through MR imaging.

\section{BEHAVIORAL TASKS \\ Setup}

The monkeys sat in a primate chair with their head fixed under dim illumination and viewed a computer monitor positioned 60 or $68 \mathrm{~cm}$ away. Eye movements were monitored during the experiments; the monkeys were trained to keep their gaze on a white fixation target, $0.2^{\circ}$ in size. While maintaining fixation, visual stimuli were presented on the screen. Eye position was monitored using an infrared eye position tracking system (model RK-716; ISCAN, Burlington, MA, USA). Eye position was sampled at $240 \mathrm{~Hz}$, digitized, and recorded. Breaks in fixation exceeding a $2^{\circ}$ window terminated the trial. Correct completion of a trial resulted in a liquid reward. In-house software (Meyer and Constantinidis, 2005) controlled the visual stimulus presentation, online monitoring of eye position, and synchronization of stimuli with neurophysiological data. The system was implemented in MATLAB (Mathworks, Natick, MA, USA), using the Psychophysics Toolbox (Brainard, 1997; Pelli, 1997). Two behavioral tasks that required working memory were used, a Match/Nonmatch task and a Delayed Match to Sample Task.

\section{Match/Nonmatch task}

Two of the monkeys were tested in the Match/Nonmatch task. The task required animals to remember the spatial location of a stimulus flashed briefly on the screen, to observe a second stimulus, and to indicate whether the two stimuli appeared at the same or different locations (Figure 2A). Trials began with the appearance of the fixation point on which the animals needed to fixate throughout the trial. This was followed by presentation of the cue, which consisted of a $2^{\circ}$ white square that could appear at one of nine locations on a $3 \times 3$ grid of $10^{\circ}$ spacing between adjacent stimuli. The position of the cue varied randomly from trial to trial so that the monkey could not predict its location. The cue was presented for $0.5 \mathrm{~s}$, and was followed by a delay period of $1.5 \mathrm{~s}$, during which only the fixation point was visible on the screen. Then a second stimulus was presented for $0.5 \mathrm{~s}$, either at the same location as the cue, constituting a match, or at the diametric location, constituting a nonmatch. This was followed by a second delay period of $1.5 \mathrm{~s}$. At the end of this interval, the fixation point disappeared and two Choice Targets appeared, consisting of a green and a blue square. The Choice Targets appeared at locations orthogonal to the Cue and Nonmatch and the location of the blue and green target randomly varied from trial to trial. If the second stimulus matched the location of the cue, the animals were trained to make an eye movement to the green target and maintain fixation for $0.5 \mathrm{~s}$; if the second stimulus was a nonmatch, then the animals were required to saccade to the blue target in order to receive a reward. Typically 10 repetitions of each cue-stimulus (match or nonmatch) combination were collected during the neurophysiological recordings.

\section{Delayed match-to-sample task}

Another two monkeys were tested with the Delayed Match-toSample Task. This task required the monkeys to remember the location of an initial stimulus, ignore stimuli appearing at other locations, and release a lever when a stimulus appeared at the location of the first stimulus (Figure 2B). The trial started with the animals pulling a behavioral lever and foveating the fixation point. Then the cue stimulus was displayed at one of nine locations, arranged along a $3 \times 3$ grid of $10^{\circ}$ separation between adjacent stimuli. For a few sessions, grid sizes of $15^{\circ}$ were used. Stimuli consisted of green or red squares of $1.5^{\circ}$ size. The color of the stimulus was the same for each neuron studied and had no significance for this experiment. The cue presentation lasted for $0.5 \mathrm{~s}$ and was followed by a delay period of $1.0 \mathrm{~s}$. A pseudorandom sequence of $0-2$ nonmatch stimuli followed, each lasting $0.5 \mathrm{~s}$ and being separated by delay periods of $0.5 \mathrm{~s}$. The sequence was completed by a match stimulus appearing at the same location as the cue. The monkeys were trained to wait for the full presentation of the match stimulus (lasting $0.5 \mathrm{~s}$ ) and to release the lever within $0.5 \mathrm{~s}$ after that in order to receive a reward. Release of the lever at any other time during the trial constituted an error and immediately aborted the trial.

The two animals used in this experiment were additionally trained in variants of the Delayed-Match-to-Sample task, in which the fixation point moved during the trial and the animals were required to match the location of the cue in either screen-centered or retinal coordinates (Rawley and Constantinidis, 2010). Only data from blocks of trials of the basic task (Figure 2B) were analyzed for the current study.

\section{SURGERY AND NEUROPHYSIOLOGY}

After the animals were trained to perform the tasks, they were prepared for neurophysiological recordings. A 20-mm diameter craniotomy was performed over the prefrontal and parietal cortex, and two recording cylinders were implanted. The location of recording cylinders was assessed with anatomical MRI imaging. Neurophysiological recordings were performed with either single or multiple microelectrodes. We used glass-coated, tungsten electrodes of $250-\mu \mathrm{m}$ diameter, with an impedance of $1 \mathrm{M} \Omega$ at $1 \mathrm{kHz}$ 
(Alpha-Omega Engineering, Nazareth, Israel) and epoxylite-coated tungsten electrodes with a diameter of $125 \mu \mathrm{m}$ and an impedance of $4 \mathrm{M} \Omega$ at $1 \mathrm{KHz}$ (FHC Bowdoin, ME, USA). Arrays of up to 8 -microelectrodes spaced $0.2-1.5 \mathrm{~mm}$ apart were advanced into the cortex through the dura with a microdrive system (EPS drive, Alpha-Omega Engineering, Nazareth, Israel). The electrical signal from each electrode was amplified, band-pass filtered between 500 and $8 \mathrm{kHz}$, and recorded with a modular data acquisition system (APM system, FHC, Bowdoin, ME). Waveforms that exceeded a user-defined threshold were sampled at $25-\mu$ s resolution, digitized and stored for off-line analysis.

\section{DATA ANALYSIS}

Action-potential waveforms recorded from microelectrodes were sorted into separate units using an automated cluster analysis method based on the KlustaKwik algorithm (Harris et al., 2000). The method relied on principal component analysis of the waveforms and was implemented in MATLAB. Firing rate of units was then determined for each of the task epochs.

We focused our analysis on neurons that responded to the visual stimuli, evidenced by significantly elevated firing rate in the 0.5 -s interval of a stimulus presentation, compared to the 0.5 or 1 -s interval of fixation (paired $t$-test; $p<0.05$ ). The spatial tuning of visually responsive neurons was assessed by comparing the discharge rates during the presentation of single stimuli at the nine grid locations. Neurons with significantly different responses to the nine stimulus locations (ANOVA; $p<0.05$ ) were included in further analysis. We tested whether a neuron exhibited persistent activity in the delay period following a stimulus by comparing its discharge rate during the delay period with its rate during the baseline, fixation period. Neurons were deemed to be exhibiting persistent responses if they displayed significantly different responses between fixation and delay period as judged by a paired $t$-test, $(p<0.05)$.

Population responses were evaluated by averaging discharges from multiple neurons and constructing population Peri-Stimulus Time Histograms. To compare responses recorded from the two brain areas and from the different tasks, we first averaged the raw firing rates from each population and then we normalized this averaged firing rate by the average firing rate recorded during the fixation period that preceded the cue presentation.

We distinguished between the patterns of delay period activity depending on whether the firing rate increased during the stimulus presentation period or not, comparing the firing rate at the beginning and end of the delay period at the location that elicited the best delay-period response. Neurons whose firing rate after the offset of the cue remained at the same levels or declined by the end of the delay period were classified as "sustained". Neurons whose firing rate increased during these time points were classified as "anticipatory".

\section{RESULTS}

\section{DATABASE}

Neuronal activity was recorded from area $7 \mathrm{a}$ of the posterior parietal cortex and areas 8 and 46 of the dorsolateral prefrontal cortex of four monkeys (Figure 1). Anatomical localization of penetrations was aided by MRI imaging performed after implantation of the recording cylinders. For each of the two tasks used, we

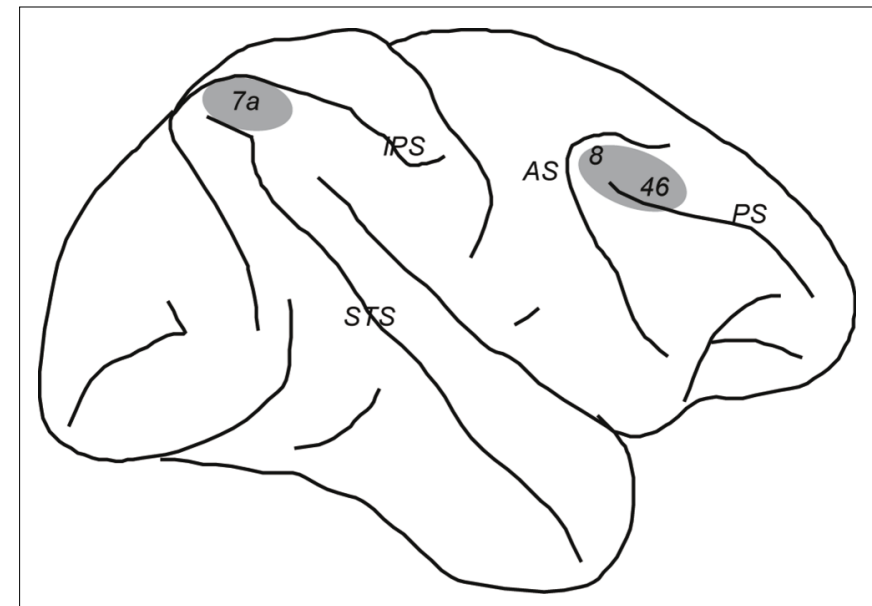

FIGURE 1 | Schematic diagram of the monkey brain. Areas were the recordings were performed are highlighted in gray. Abbreviations: AS, Arcuate Sulcus; IPS, Intraparietal Sulcus; PS, Prinsipal Sulcus; STS, Superior Temporal Sulcus.

selected a sample of neurons that a) responded to visual stimuli, b) displayed significant selectivity for the spatial location of the stimuli (ANOVA, $p<0.05$ ), allowing us to identify locations in and out of their receptive fields, and c) exhibited significantly elevated discharges in the delay period after the offset of the cue stimulus compared to the baseline period (paired $t$-test, $p<0.05$ ). We used these neurons to examine how responses varied following a second stimulus presentation in or out of the receptive field (although the activity recorded in the second delay period was not a selection criterion).

Two monkeys were tested with the Match/Nonmatch task (Figure 2A). We recorded from 149 neurons in the dorsolateral prefrontal cortex that responded to visual stimuli $(t$-test, $p<0.05)$ and exhibited selectivity for the spatial location of the cue (ANOVA, $p<0.05$ ). Of those neurons, 83 exhibited significantly elevated activity during the first delay period after the cue presentation $(t$ test, $p<0.05)$ and were tested at locations inside and out of the receptive fields. We also recorded from 60 visually responsive and spatially selective neurons in the posterior parietal cortex of the same animals. Of those, 38 exhibited delay period activity and were tested at locations inside and out of the receptive fields. These 83 and 38 neurons respectively make up the sample under study from the Match/Nonmatch task.

Two different monkeys were tested with the spatial version of the Delayed Match-to-Sample task (Figure 2B). In this experiment, we recorded from 148 visually responsive and spatially selective neurons in the dorsolateral prefrontal cortex. Of those, 48 neurons exhibited delay period activity and were tested at locations inside and out of the receptive fields. Finally, we recorded from 119 visually responsive and spatially selective neurons in the posterior parietal cortex, 36 of which also exhibited delay period activity and were tested at locations inside and out of the receptive fields. Analysis was performed on this sample of 48 and 36 neurons from the Delayed Match-to-Sample task. The total number of neurons included in our sample, broken down by task, monkey, brain area and response type (explained in the next section) are shown in Table 1. 
A Match - Nonmatch Task

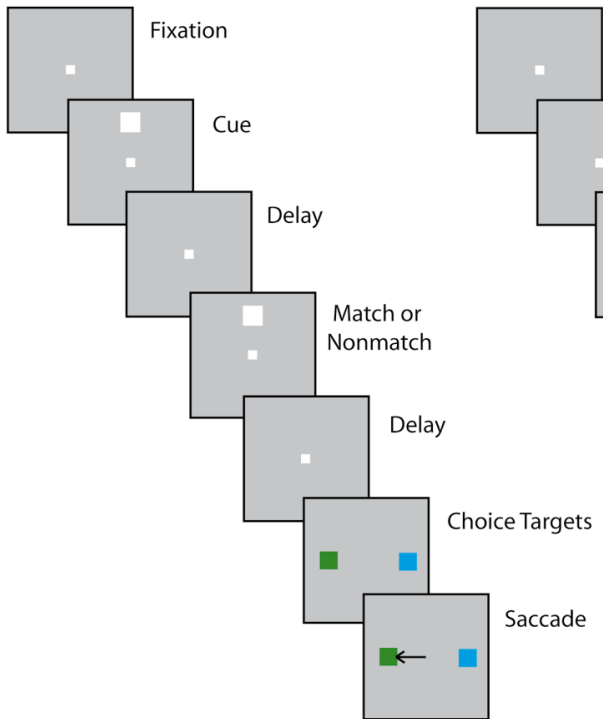

B Delayed Match-to-Sample Task

Fixation

Cue

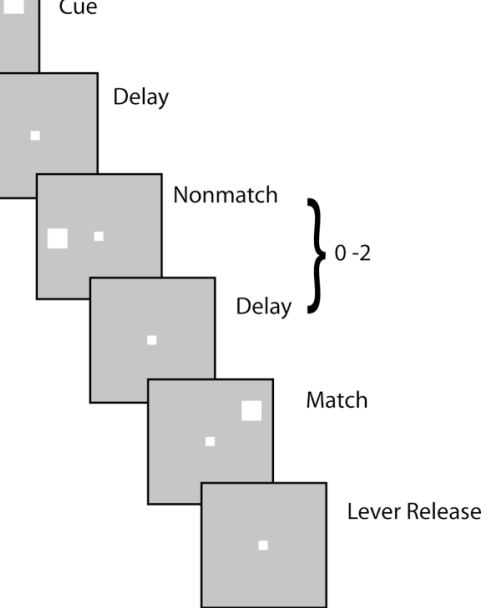

FIGURE 2 | Behavioral tasks. Successive frames indicate the series of stimulus presentations. (A) Stimulus presentations in the Match/Nonmatch task. (B) Stimulus presentations in the Delayed Match-to-Sample task.

Table 1 | Number of neurons recorded in each area and monkey.

\begin{tabular}{lllll}
\hline Task & Monkey & Area & Sustained & Anticipatory \\
\hline Match-Nonmatch & EL & PFC & 42 & 23 \\
& & PPC & 19 & 19 \\
& AD & PFC & 16 & 2 \\
& & PPC & 0 & 0 \\
& Total & PFC & 58 & 25 \\
Delayed match & & PPC & 19 & 19 \\
to sample & CA & PFC & 1 & 0 \\
& & PPC & 2 & 0 \\
& DA & PFC & 37 & 10 \\
& & PPC & 15 & 19 \\
& Total & PFC & 38 & 10 \\
& & PPC & 17 & 19
\end{tabular}

\section{TYPES OF DELAY PERIOD ACTIVITY}

By examining the time course of delay period activity, we found that neurons exhibited one of two different types of responses, as it has also been described in previous studies (Quintana and Fuster, 1992; Constantinidis and Steinmetz, 1996). One class of neurons exhibited persistent responses with a fairly constant or slightly declining rate, which appeared to extend a response to the cue presentation; we refer to these as sustained responses (Figure 3). Across the two tasks, 96 prefrontal neurons and 36 parietal neurons were classified as exhibiting sustained activity. Another class of neurons started to discharge after the offset of the cue, with a rate that accelerated during the delay period; we refer to these as anticipatory responses (Figure 4). The strongest delay-period anticipatory responses were often observed in the delay period following a cue presentation out of the receptive field (Figure 4C). A total of 35 prefrontal and 38 parietal neurons were classified as anticipatory.

\section{DELAY PERIOD ACTIVITY IN THE MATCH/NONMATCH TASK}

We first examined the responses of prefrontal and parietal neurons in the Match/Nonmatch Task. In this task, the animals were required to remember the location of the cue stimulus and to decide if the second stimulus appeared in the same or different location (Figure 2A). We sought to determine if activity in the delay periods differed after the presentation of a stimulus in and out of the receptive field. Examples of neurons with sustained activity recorded from the posterior parietal and dorsolateral prefrontal cortex are shown in Figures 3 and 5, respectively. In agreement with previous studies, we found that posterior parietal neurons that exhibited sustained responses after a cue presentation in the receptive field, typically ceased to respond after presentation of a subsequent nonmatch stimulus out of the receptive field (Figure 3B). In contrast, neurons in the prefrontal cortex during execution of the same task continued to discharge even after a transient decrease in activity caused by the nonmatch stimulus (Figure 5B).

These patterns of activity were evident on the population response as well. The population of both prefrontal and parietal neurons responded robustly with sustained activity above the baseline in both delay periods in trials of cue and match stimuli appearing in the receptive field (Figure 6A). More importantly, in trials where the cue appeared in the receptive field but was followed by a stimulus out of the receptive field, prefrontal neurons continued to discharge in a robust manner in the second delay period whereas the activity of parietal neurons returned to baseline (Figure 6B), which constituted a significant difference between the two areas ( $t$-test, $p<0.05$ ).

We further examined the phenomenon on a neuron-by-neuron basis, by plotting the firing rate we observed in two conditions. Each data point in Figure 7 represents the firing rate of a single neuron during the delay period following presentation of a nonmatch stimulus out of the receptive field (as in Figure 6B) as a function of the firing rate observed in the delay period after presentation 


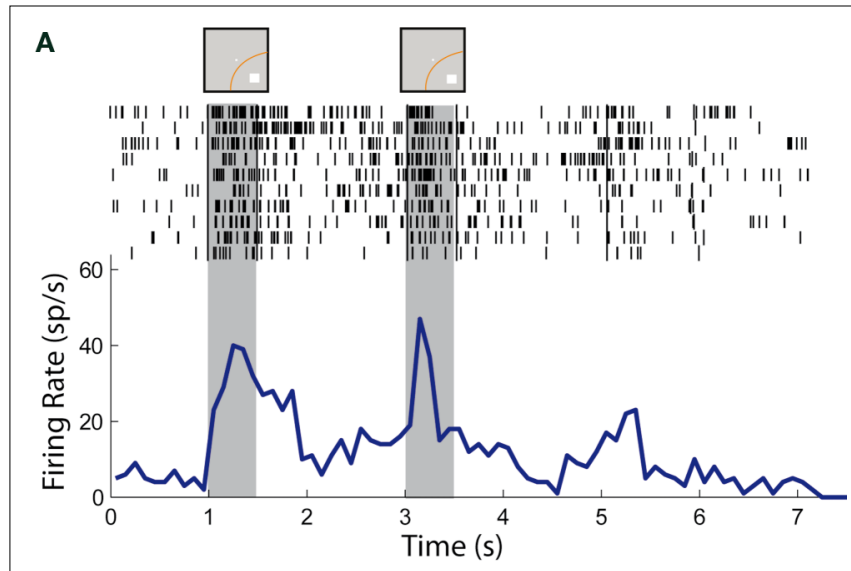

B
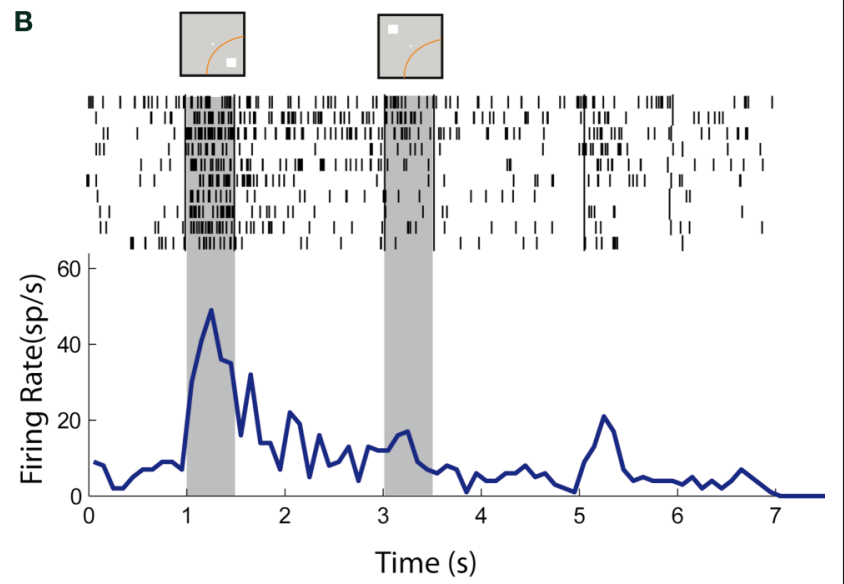

C
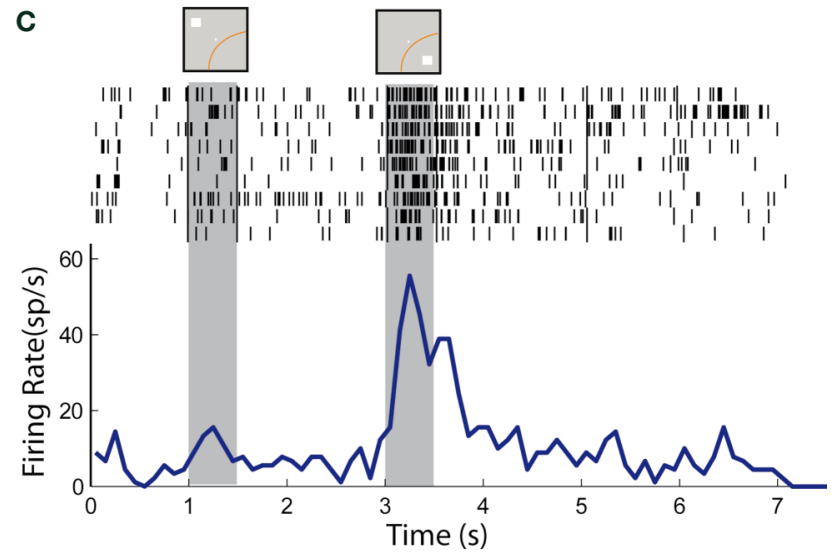

FIGURE 3 | Rasters and histograms of one neuron with sustained activity recorded from area 7a of the posterior parietal cortex. Gray bars represent times of stimulus presentations. Insets on top of gray bars indicate stimulus location relative to the receptive field (orange curve). Two sets of vertical lines in the rasters represent onset of Choice Targets and delivery of reward.

(A) Responses to the cue followed by a match stimulus in the receptive field.

(B) Responses to the cue in the receptive field followed by a nonmatch stimulus out of the receptive field. (C) Responses to the cue out of the receptive field, followed by a nonmatch in the receptive field.

of a match stimulus in the receptive field (as in Figure 6A). A regression analysis indicated that for prefrontal neurons these responses were essentially equal (regression slope $=1.03$, practically coinciding with the diagonal). Parietal neurons responded

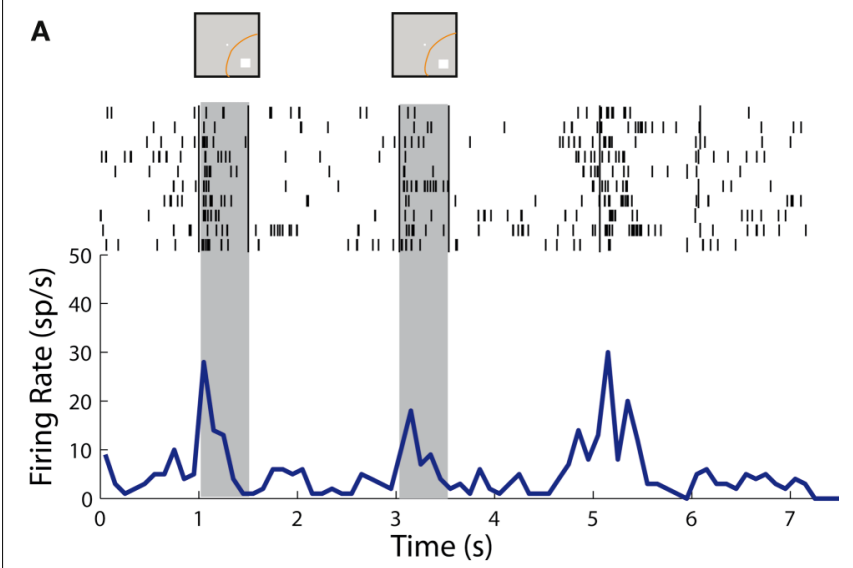

B
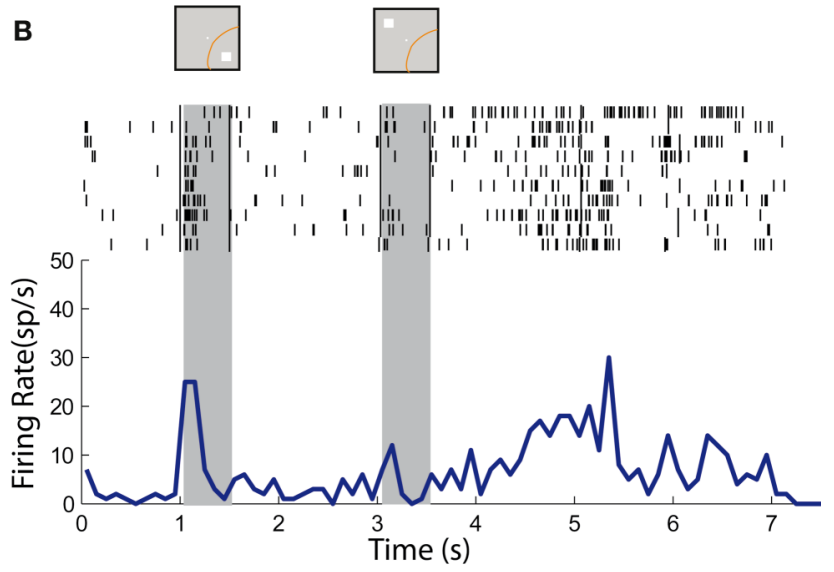

C

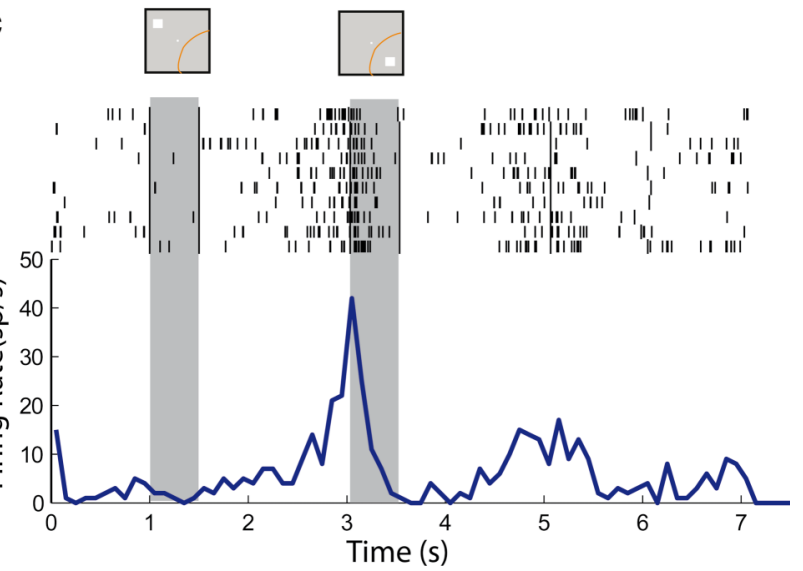

FIGURE 4 | Rasters and histograms of one neuron with anticipatory activity recorded from area $7 \mathrm{a}$ of the posterior parietal cortex.

Conventions are the same as in Figure 3. (A,B,C) Responses to the cue followed by a stimulus in the receptive field $(\mathbf{A}, \mathbf{C})$ or out of the receptive field (B), as in Figure 3.

with consistently lower firing rates after nonmatch stimuli appeared out of the receptive field (regression slope $=0.58$ ). The slopes of the two regression lines were significantly different from each other $(F$-test, $p<0.05)$.

On the other hand, we saw examples of both parietal neurons and prefrontal neurons that continued to display sustained discharges after the appearance of the second stimulus in the 


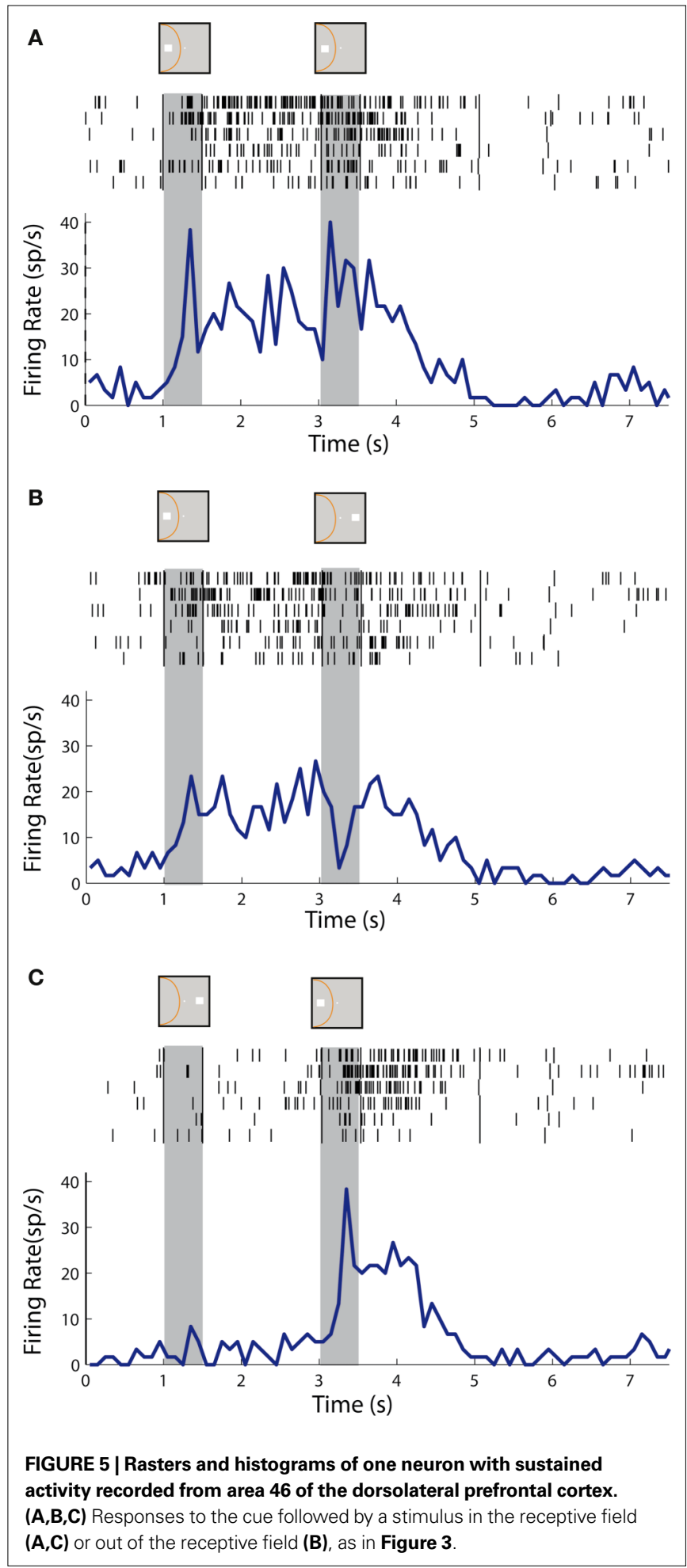

receptive field, when the initial cue appeared out of the receptive field (Figures 3C and 5C, respectively). This was also evident in the population responses from the two areas (Figure 6C). We should point out that the nonmatch stimulus was behaviorally relevant in this task; the animals were required to consider both stimuli and make a decision on whether they matched each other or not. Our

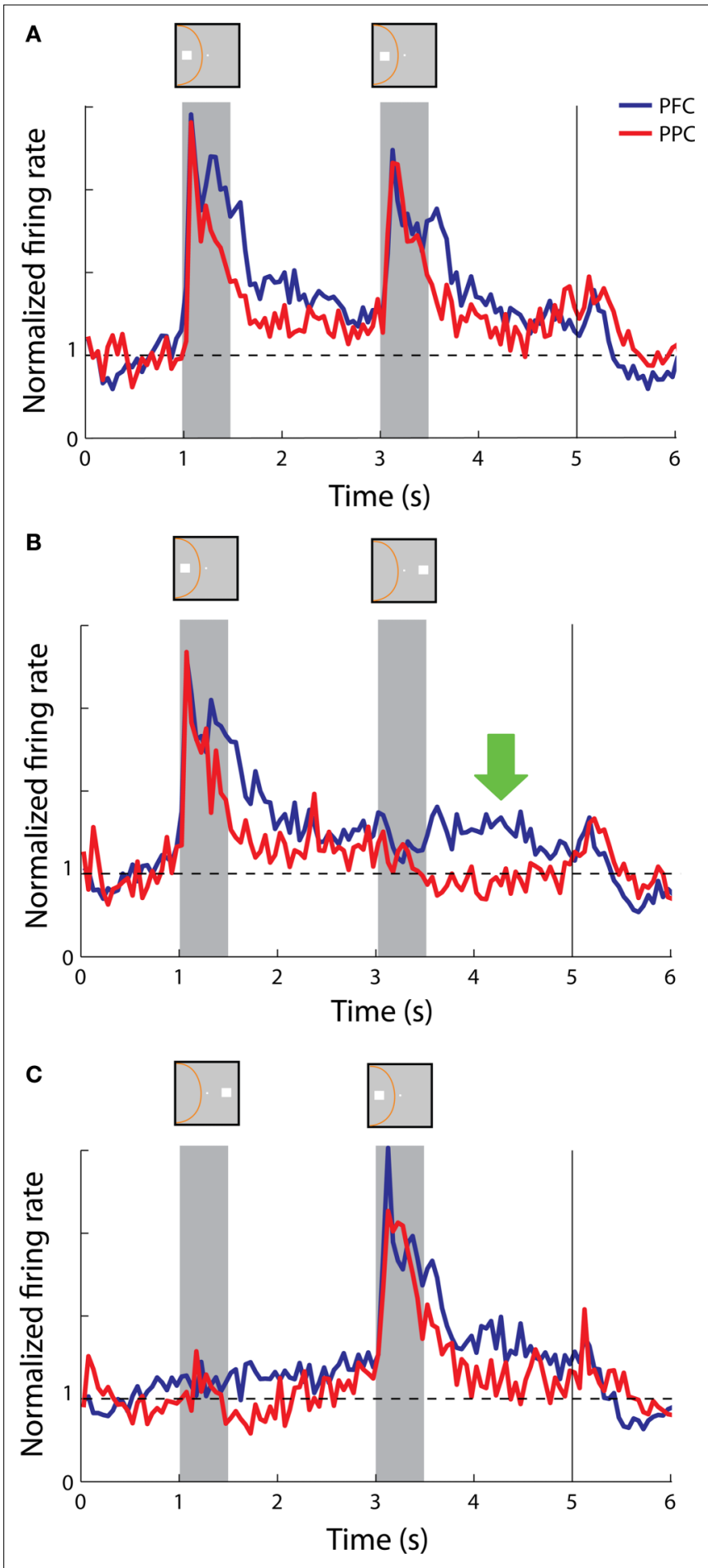

FIGURE 6 | Population responses from neurons with sustained activity recorded in the prefrontal $(N=58)$ and posterior parietal cortex $(N=19)$. Vertical line represents onset of Choice Targets. Green arrow represents the delay period following a nonmatch stimulus out of the receptive field. Data are shown from the Match/Nonmatch task. (A) Average, normalized responses to the cue followed by a match stimulus in the receptive field. Stimulus and receptive field locations drawn for illustration purposes - the actual locations differed for each neuron. (B) Average responses to the cue in the receptive field followed by a nonmatch stimulus out of the receptive field. (C) Average responses to the cue out of the receptive field, followed by a nonmatch stimulus in the receptive field. 


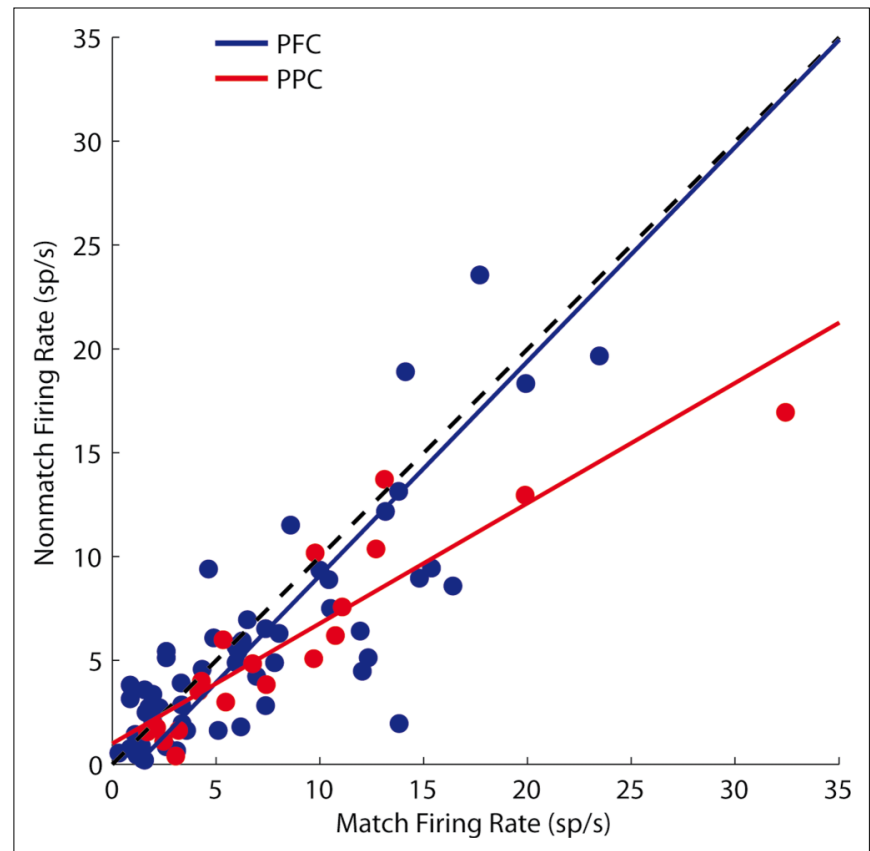

FIGURE 7 | Comparison of responses in the second delay period following a match and nonmatch stimulus, when the cue appeared in the receptive field. Each dot represents a single neuron. One outlier is omitted. Regression lines are shown for the prefrontal and posterior parietal cortex.

findings show that both prefrontal and parietal neurons continued to represent a previous stimulus that appeared in their receptive field, even though the cue appeared outside.

Anticipatory activity averaged across the population of neurons was generally not informative about the location of the cue or preceding stimulus, in or out of the receptive field. Individual neurons exhibited a wide range of response envelopes but we observed stronger anticipatory activity in the first delay period when it followed a cue presentation out of the receptive field (Figure 8C) than a cue in the receptive field (Figures 8A,B). We also observed robust anticipatory activity in the second delay period in both the prefrontal and posterior parietal cortex whether the second stimulus was a match in the receptive field, a nonmatch out of the receptive field, or a nonmatch in the receptive field (Figure 8). Although weaker, anticipatory activity was also evident even before the appearance of the cue (Figure 8, time interval 0-1 s).

\section{DELAY PERIOD ACTIVITY IN THE DELAYED MATCH-TO-SAMPLE TASK}

We repeated our analysis of delay period activity in the Delayed Match-to-Sample task. In this task, the animals were required to remember the location of the cue, to ignore a random number of nonmatch stimulus presentations, and to release a lever when a match stimulus appeared at the same location as the cue (Figure 2B). We again distinguished between sustained and anticipatory responses. As was the case for the Match/Nonmatch task, the strongest anticipatory responses were not aligned with the presentation of the cue in the receptive field (data not shown). We therefore focused on sustained responses and we sought to determine if responses during the delay period differed depending on whether the cue and nonmatch stimuli appeared in or out of the receptive field.
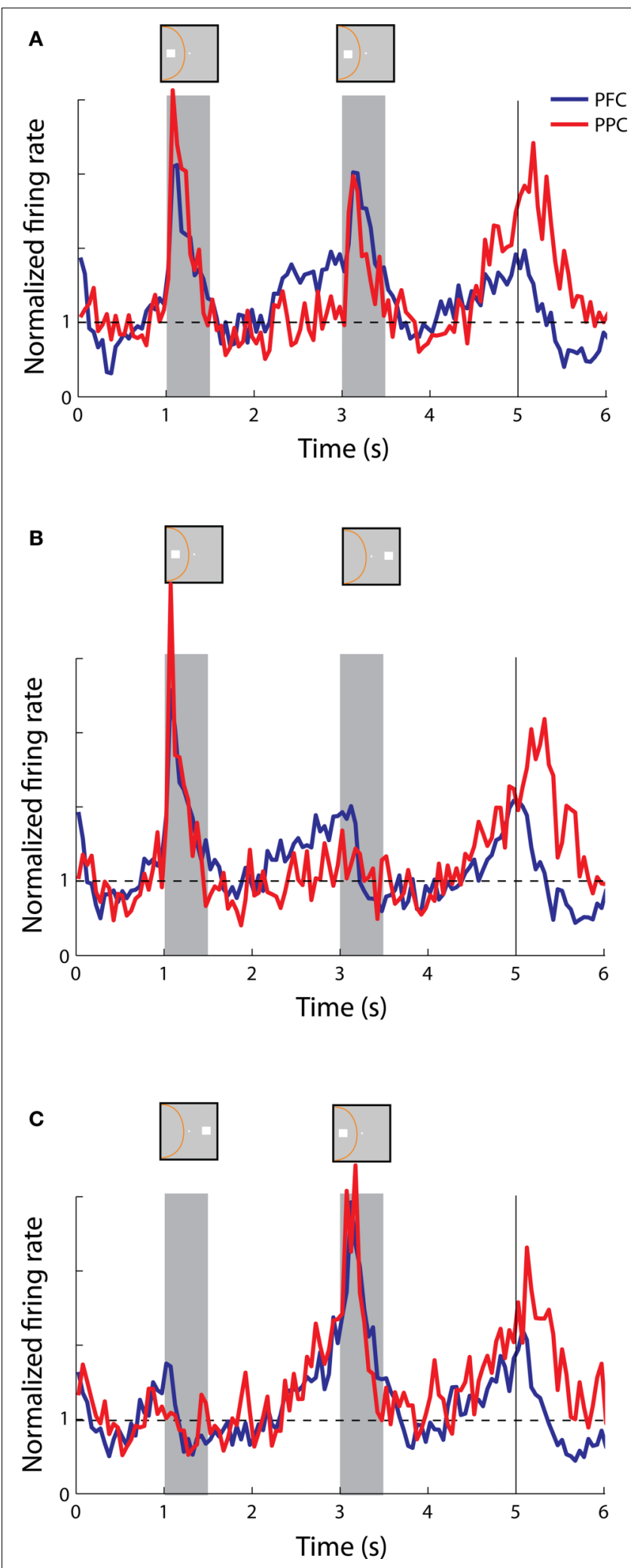

FIGURE 8 | Population responses from neurons with anticipatory activity recorded in the prefrontal $(N=25)$ and posterior parietal cortex $(N=19)$. $(\mathbf{A}, \mathbf{B}, \mathbf{C})$ Average, normalized responses to the cue followed by a stimulus in the receptive field $(\mathbf{A}, \mathbf{C})$ or out of the receptive field $(\mathbf{B})$, as in Figure $\mathbf{6}$. 


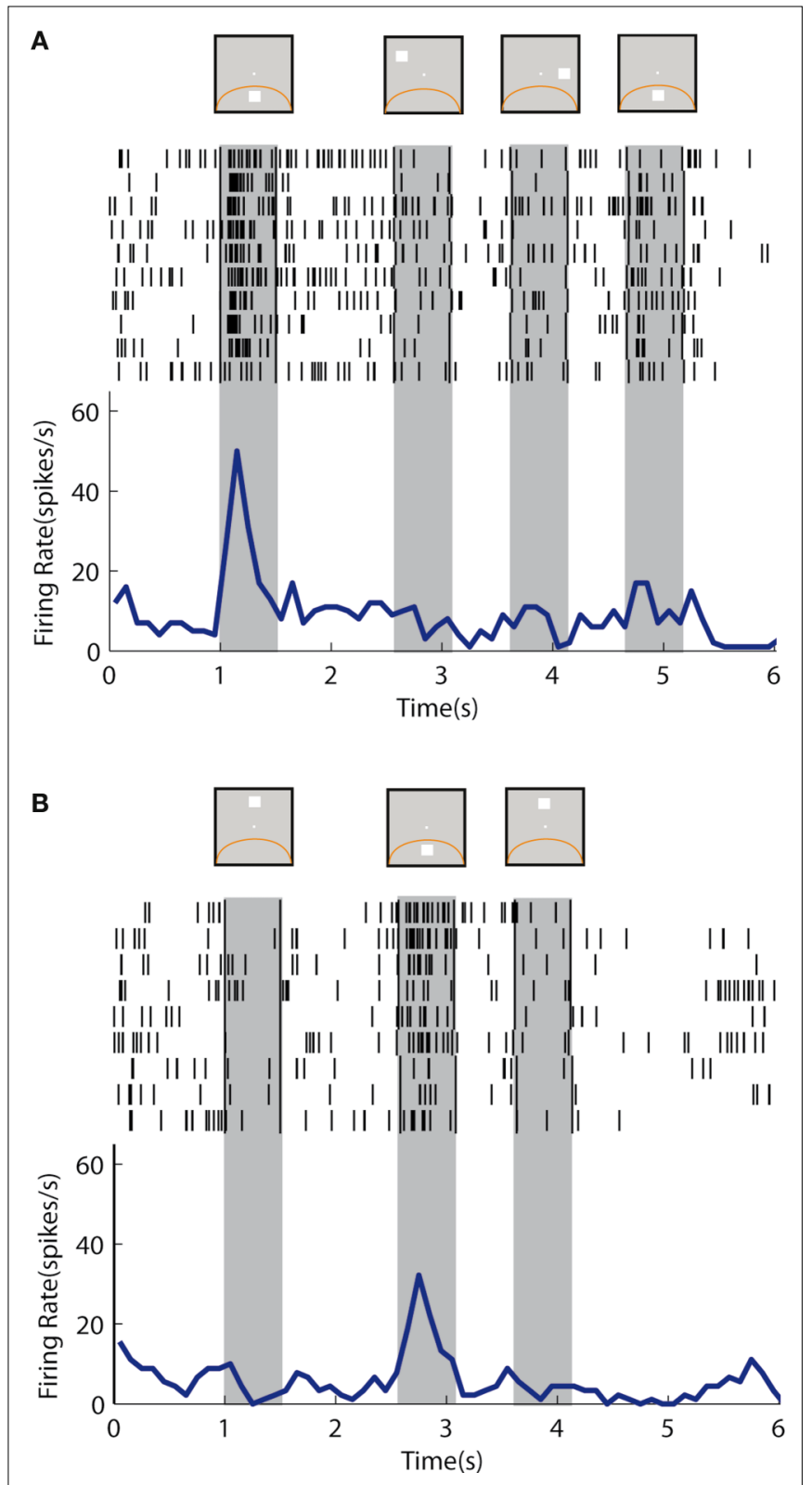

FIGURE 9 | Rasters and histograms of one neuron with sustained activity recorded from area $7 \mathrm{a}$ of the posterior parietal cortex, tested with the delayed match-to-sample task. Data are shown from the Match/Nonmatch task. (A) Responses to a cue presentation in the receptive field followed by nonmatch presentations out of the receptive field. (B) Responses to a cue presentation out of the receptive field followed by a nonmatch stimulus in the receptive field.

An example neuron with sustained activity recorded from the posterior parietal cortex is shown in Figure 9. The neuron responded with sustained activity after presentation of the cue in the receptive field, which was diminished after presentation of a nonmatch stimulus out of the receptive field (Figure 9A). The same neuron was activated by a nonmatch stimulus that appeared in the receptive field thought that was not followed by appreciable delay period activity (Figure 9B). A neuron recorded from the prefrontal cortex is shown in Figure 10.

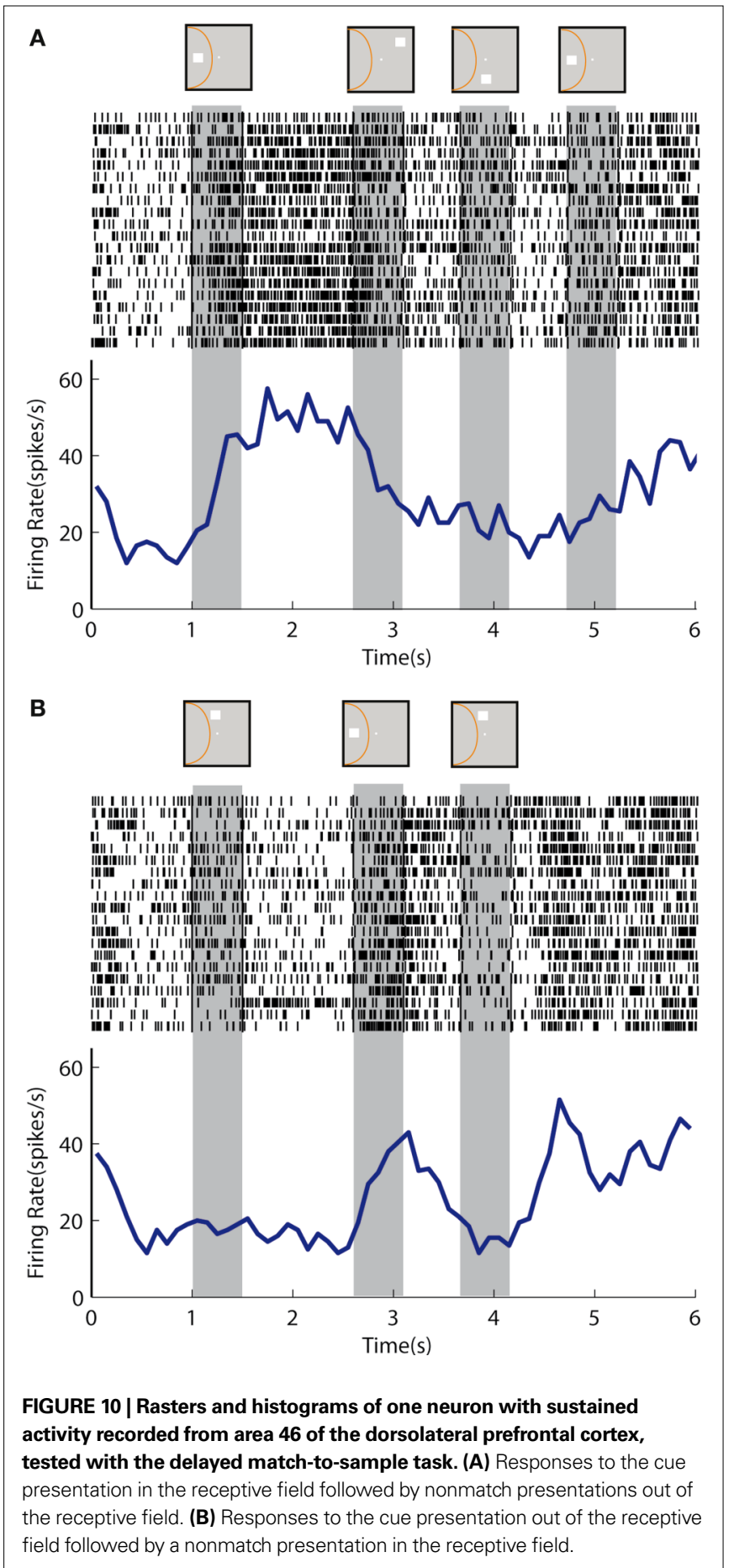

The neuron exhibited sustained responses after the cue presentation in the receptive field, which declined after presentation of nonmatch stimuli out of the receptive fields (Figure 10A). This neuron also responded to a nonmatch stimulus in the receptive field and continued to discharge in the delay period, until a match stimulus out of the receptive field was presented (Figure 10B). Activity after presentation of the last stimulus is associated with the lever movement, release of fixation control and reward delivery. 


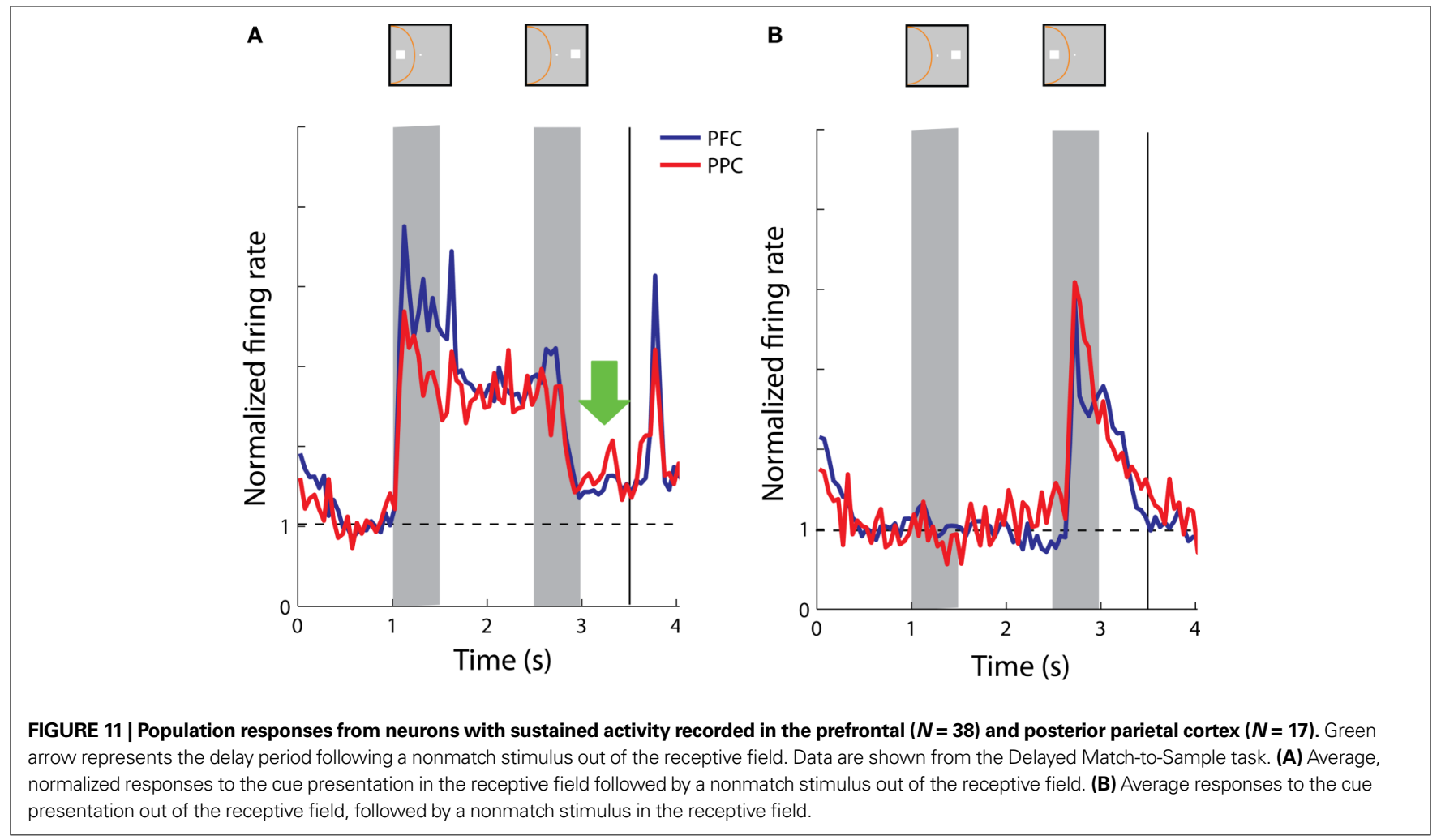

We again constructed population histograms of delay period activity and investigated whether sustained activity following a cue presentation in the receptive field was interrupted by a stimulus out of the receptive field and vice versa. Note that the match stimulus terminates the trial (the monkey releases the lever) and for this reason the period after the match stimulus is not informative and is not shown in Figure 11, unlike Figure 6. When we compared sustained responses in the delay period following a nonmatch stimulus out of the receptive field across the populations of parietal and prefrontal neurons, we found no significant differences $(t$-test, $p>0.2)$ between average response rates in the two areas (Figure 11A). The same was true for activity following a nonmatch stimulus in the receptive field, when the cue appeared out of the receptive field; both prefrontal and posterior parietal neurons continued to be active in the second delay period (Figure 11B).

\section{DISCUSSION}

\section{OVERVIEW OF THE FINDINGS}

Our study revealed differences between the patterns of delay period activity among prefrontal and parietal neurons in a spatial working memory task. These became clear when we distinguished between sustained and anticipatory responses. Distinct patterns of responses were observed in the populations of neurons with sustained responses which exhibited differences in the prefrontal vs. the parietal cortex (Figure 6). Anticipatory responses with similar time courses were observed in the two areas (Figure 8). Our main finding was that dorsolateral prefrontal activity representing the spatial location of a cue survives the presentation of a nonmatch stimulus out of the receptive field in the context of a Match/Nonmatch task (Figure 6B).
At the same time, we found considerable similarities between the regions. Both prefrontal and parietal neurons continued to be active after the presentation of a nonmatch stimulus in the Match/ Nonmatch task (Figures 3C and 5C). We also found responses of prefrontal and parietal neurons to be quite similar in the context of the Delayed Match-to-Sample task. Although we observed individual examples of prefrontal neurons that were able to resist the appearance of multiple nonmatch stimuli and continue to discharge in response to an initial cue stimulus (Figure 10B), overall the populations of prefrontal and parietal neurons exhibited delay period activity that was diminished but not extinguished after a nonmatch stimulus out of the receptive field (Figure 10A). Similarly, both prefrontal and parietal neurons continue to represent a nonmatch stimulus in the receptive field in the delay period following it, although the monkeys were required only to remember the location of the cue in this task (Figure 10B).

\section{TYPES OF RESPONSES}

Analysis of our data set identified two general types of neuronal activity in the delay period, in agreement with previous studies (Quintana and Fuster, 1992; Constantinidis and Steinmetz, 1996). Sustained activity was characterized by persistent discharges that extended beyond the initial response to a stimulus into the delay period and could encode the location of the preceding stimulus. This is the type of activity commonly thought to provide a neural correlate of working memory for the preceding stimulus (Goldman-Rakic, 1995).

Anticipatory activity tended to appear after presentation of a stimulus at any spatial location, most often outside the neuron's receptive field. This type of activity is commonly observed in 
neurophysiological recordings in the prefrontal cortex (Rainer and Miller, 2002; Brody et al., 2003) however its properties are not as straightforward. Previous studies have shown that anticipatory activity after a stimulus presentation is not simply related to expectation about the appearance of a subsequent stimulus, but it is in fact tuned depending on the location of the preceding stimulus (Constantinidis and Steinmetz, 1996). This was the case even when the location of the subsequent stimulus was randomized, making it impossible for the monkey to truly anticipate its location. In sum, anticipatory activity can be informative about the location of the preceding stimulus, however it displays a tuning that differs from the tuning observed during the stimulus presentation. Recent computational studies have shown that firing rates of individual prefrontal neurons exhibit a wide variety of time courses, with memory-related activity components accounting only for a small percentage of the total variance (Machens et al., 2010). In our experiments, prefrontal and posterior parietal neurons exhibited very similar patterns of anticipatory activity that could not distinguish the two areas.

In recent years, mechanisms of memory maintenance in the absence of increased firing rate in the delay period activity have also been described (Sugase-Miyamoto et al., 2008). Modeling studies have demonstrated that synaptic changes in the absence of increased firing rate can account for such phenomena (Mongillo et al., 2008).

\section{PREVIOUS COMPARISONS OF PARIETAL-PREFRONTAL ACTIVITY}

An earlier study directly comparing prefrontal and parietal activity in the oculomotor delayed response (ODR) task observed remarkable similarities between neurons in the two regions (Chafee and Goldman-Rakic, 1998). The ODR task involves presentation of a single stimulus, followed by a delay period, and the animals are required to execute an eye movement towards the location of the remembered cue. Data from other experimental paradigms that involved presentation of multiple stimuli only one of which the animals were required to remember suggest differences between regions (di Pellegrino and Wise, 1993; Constantinidis and Steinmetz, 1996). It should be clear however that comparison between results from those studies is not straightforward since the corresponding studies involved different task and behavioral paradigms, conducted in different laboratories. Our current results provide the first direct distinction of prefrontal and parietal responses in the same animals, while performing the same task (Figure 6).

\section{TASK EFFECTS}

The two behavioral tasks that we used in this study place different demands on memory. The Match/Nonmatch task requires the animals to remember the initial stimulus (cue) and to compare it with a second stimulus presentation (match or nonmatch). At the end of the second stimulus presentation, the animals no longer need to remember either stimulus and simply have to decide on whether the two stimuli matched each other or not. Nonetheless, we observed responses in the second delay period that continued to represent either the initial or second stimulus. A similar representation of a previously presented stimulus has been described for tactile stimuli (Romo et al., 1999, 2002). In the task used by Romo et al., monkeys were presented with two vibratory stimuli in sequence separated by delay periods. At the end of the trial, the animals were required to decide if the second frequency was lower or higher than the first. As in our task, activity of some prefrontal neurons continued to represent stimulus attributes in the second delay period. We have recently observed responses in the prefrontal cortex that continue to track a visual stimulus, even in the complete absence of a memory requirement, in animals only trained to fixate (Meyer et al., 2007). A similar effect, albeit using a much shorter delay period, was also observed in the posterior parietal cortex (Joelving et al., 2007). These results suggest that prefrontal and parietal neurons continue to track properties of physical stimuli even when they are not required to do so by a behavioral task.

The choice of task was critical in revealing differences between the two areas. As was the case with the use of the Oculomotor Delayed Response task (Chafee and Goldman-Rakic, 1998), we observed no significant differences in the averaged prefrontal and parietal responses in the context of the Delayed Match To Sample Task. This finding indicates that computational power related to the maintenance of spatial information is considerably distributed between the prefrontal and posterior parietal cortex and differences between the two areas are task dependent.

\section{IMPLICATIONS FOR FUNCTIONAL SPECIALIZATION}

The posterior parietal and dorsolateral prefrontal cortexes are strongly interconnected (Cavada and Goldman-Rakic, 1989) and share many functional properties. Our current results indicate that the prefrontal cortex has unique properties in its ability to preserve information in working memory even after the presentation of additional stimuli, at least in the context of some behavioral tasks. What intrinsic properties of the prefrontal cortex give rise to this unique ability is largely an open question although many candidates have been identified. Computational models have demonstrated that dopamine can serve to stabilize working memory (Durstewitz et al., 2000). This is thought to occur through an increase of NMDA conductance (Yang and Seamans, 1996; Seamans et al., 2001; Chen et al., 2004), which can facilitate persistent activity by its slow time constant, leaving the postsynaptic neuron in a depolarized state for a longer time interval (Wang, 2001). The prefrontal cortex, unlike the posterior parietal cortex, receives a significant dopaminergic innervation from the ventral tegmental area, which could account for the physiological differences. NMDA receptor density itself may be a factor in the ability of cortical neurons to resist interference (Compte et al., 2000) and evidence suggests that prefrontal neurons may be characterized by unique patterns of NMDA activation (Wang et al., 2008). Other differences in the intrinsic circuits of the prefrontal and parietal cortex in terms of dendritic tree size and numbers of synapses may also be contributing factors; prefrontal pyramidal neurons exhibit the most extensive dendritic trees and highest number of spines of any cortical neurons (Elston, 2000, 2003). Finally, the relative composition of interneuron types may be different for the prefrontal cortex (Wang et al., 2004). It appears likely that a combination of factors endows the prefrontal cortex with unique properties, such as those we observed in our neurophysiological recordings.

\section{ACKNOWLEDGMENTS}

We wish to thank Keith Roberts and Kathini Palaninathan for their technical contributions to the experiments. This work was supported by NIH grants EY017077 and EY016773. 


\section{REFERENCES}

Baddeley, A. (1992). Working memory. Science 255, 556-559.

Brainard,D.H.(1997). The psychophysics toolbox. Spat. Vis. 10, 433-436.

Brody, C.D., Hernandez,A.,Zainos, A., and Romo, R. (2003). Timing and neural encoding of somatosensory parametric working memory in macaque prefrontal cortex. Cereb. Cortex 13, 1196-1207.

Cavada, C., and Goldman-Rakic, P. S. (1989). Posterior parietal cortex in rhesus monkey: I. Parcellation of areas based on distinctive limbic and sensory corticocortical connections. J. Comp. Neurol. 287, 393-421.

Chafee, M. V., and Goldman-Rakic, P. S. (1998). Matching patterns of activity in primate prefrontal area $8 \mathrm{a}$ and parietal area 7ip neurons during a spatial working memory task. J. Neurophysiol. 79, 2919-2940.

Chen, G., Greengard, P., and Yan, Z. (2004). Potentiation of NMDA receptor currents by dopamine $\mathrm{D} 1$ receptors in prefrontal cortex. Proc. Natl. Acad. Sci. U.S.A. 101, 2596-2600.

Compte, A., Brunel, N., Goldman-Rakic, P. S., and Wang, X. J. (2000). Synaptic mechanisms and network dynamics underlying spatial working memory in a cortical network model. Cereb. Cortex 10, 910-923.

Constantinidis, C., and Procyk, E. (2004). The primate working memory networks. Cogn. Affect. Behav. Neurosci. 4, 444-465.

Constantinidis, C., and Steinmetz, M. A. (1996). Neuronal activity in posterior parietal area 7a during the delay periods of a spatial memory task. $J$. Neurophysiol. 76, 1352-1355.

di Pellegrino, G., and Wise, S. P. (1993). Visuospatial versus visuomotor activity in the premotor and prefrontal cortex of a primate. J. Neurosci. 13, 1227-1243.

Durstewitz, D., Seamans, J. K., and Sejnowski, T. J. (2000). Dopaminemediated stabilization of delayperiod activity in a network model of prefrontal cortex. J. Neurophysiol. 83, 1733-1750.

Elston, G. N. (2000). Pyramidal cells of the frontal lobe: all the more spinous to think with. J. Neurosci. 20:RC95.
Elston, G. N. (2003). The pyramidal neuron in occipital, temporal and prefrontal cortex of the owl monkey (Aotus trivirgatus): regional specialization in cell structure. Eur. J. Neurosci. 17, 1313-1318.

Funahashi, S., Bruce, C. J., and GoldmanRakic, P. S. (1989). Mnemonic coding of visual space in the monkey's dorsolateral prefrontal cortex. $J$. Neurophysiol. 61, 331-349.

Fuster, J. M., and Alexander, G. E. (1971). Neuron activity related to short-term memory. Science 173, 652-654.

Goldman-Rakic, P. S. (1995). Cellular basis of working memory. Neuron 14, 477-485.

Harris, K. D., Henze, D. A., Csicsvari, J., Hirase, H., and Buzsaki, G. (2000). Accuracy of tetrode spike separation as determined by simultaneous intracellular and extracellular measurements. J. Neurophysiol. 84, 401-414.

Joelving, F. C., Compte, A., and Constantinidis, C. (2007). Temporal properties of posterior parietal neuron discharges during working memory and passive viewing. J. Neurophysiol. 97, 2254-2266.

Machens, C. K., Romo, R., and Brody, C.D. (2010). Functional, but not anatomical, separation of "What" and "When" in prefrontal cortex. J. Neurosci. 30, 350-360.

Meyer, T., and Constantinidis, C. (2005) A software solution for the control of visual behavioral experimentation. $J$. Neurosci. Methods 142, 27-34.

Meyer, T., Qi, X. L., and Constantinidis, C. (2007). Persistent discharges in the prefrontal cortex of monkeys naive to working memory tasks. Cereb. Cortex 17(Suppl. 1), i70-i76.

Miller, E. K., Erickson, C. A., and Desimone, R. (1996). Neural mechanisms of visual working memory in prefrontal cortex of the macaque. $J$. Neurosci. 16, 5154-5167.

Miller, E. K., Li, L., and Desimone, R. (1993). Activity of neurons in anterior inferior temporal cortex during a short-term memory task. J. Neurosci. 13, 1460-1478.

Mongillo, G., Barak, O., and Tsodyks, M. (2008). Synaptic theory of working memory. Science 319, 1543-1546.
Pasternak, T., and Greenlee, M.W. (2005) Working memory in primate sensory systems. Nat. Rev. Neurosci. 6 , 97-107.

Pelli, D. G. (1997). The VideoToolbox software for visual psychophysics: transforming numbers into movies. Spat. Vis. 10, 437-442.

Quintana, J., and Fuster, J. M. (1992). Mnemonic and predictive functions of cortical neurons in a memory task. Neuroreport 3, 721-724.

Rainer, G., and Miller, E. K. (2002) Timecourse of object-related neural activity in the primate prefrontal cortex during a short-term memory task. Eur. J. Neurosci. 15, 1244-1254.

Rawley, J. B., and Constantinidis, C. (2010). Effects of task and coordinate frame of attention in area $7 \mathrm{a}$ of the primate posterior parietal cortex. $J$. Vis. 10, 1-16.

Romo, R., Brody, C. D., Hernandez, A., and Lemus, L. (1999). Neuronal correlates of parametric working memory in the prefrontal cortex. Nature 399 470-473.

Romo, R., Hernandez, A., Zainos, A., Lemus, L., and Brody, C. D. (2002). Neuronal correlates of decisionmaking in secondary somatosensory cortex. Nat. Neurosci. 5, 1217-1225.

Seamans, J. K., Durstewitz, D., Christie, B. R., Stevens, C. F., and Sejnowski, T. J. (2001). Dopamine D1/D5 receptor modulation of excitatory synaptic inputs to layer $\mathrm{V}$ prefrontal cortex neurons. Proc. Natl. Acad. Sci. U.S.A. 98, 301-306.

Sigala, N. (2009). Visual working memory and delay activity in highly selective neurons in the inferior temporal cortex. Front. Syst. Neurosci. 3:11. doi: 10.3389/neuro.06.011.2009.

Sugase-Miyamoto, Y., Liu, Z., Wiener, M. C., Optican, L. M., and Richmond, B. J. (2008). Short-term memory trace in rapidly adapting synapses of inferior temporal cortex. PLoS Comput. Biol. 4:e1000073. doi:10.1371/journal. pcbi. 1000073 .

Takeda, M., Naya, Y., Fujimichi, R. Takeuchi, D., and Miyashita, Y. (2005) Active maintenance of associative mnemonic signal in monkey inferior temporal cortex. Neuron 48, 839-848.
Wang, H., Stradtman, G. G. III, Wang, X. J., and Gao, W. J. (2008). A specialized NMDA receptor function in layer 5 recurrent microcircuitry of the adult rat prefrontal cortex. Proc. Natl. Acad. Sci. U.S.A. 105, 16791-16796.

Wang, X. J. (2001). Synaptic reverberation underlying mnemonic persistent activity. Trends Neurosci. 24, 455-463.

Wang, X. J., Tegner, J., Constantinidis, C., and Goldman-Rakic, P. S. (2004) Division of labor among distinct subtypes of inhibitory neurons in a cortical microcircuit of working memory. Proc. Natl. Acad. Sci. U.S.A. 101, 1368-1373.

Woloszyn, L., and Sheinberg, D. L. (2009). Neural dynamics in inferior temporal cortex during a visual working memory task. J. Neurosci. 29, 5494-5507.

Yang, C. R., and Seamans, J. K. (1996) Dopamine D1 receptor actions in layers V-VI rat prefrontal cortex neurons in vitro: modulation of dendriticsomatic signal integration. J. Neurosci. 16, 1922-1935.

Conflict of Interest Statement: The authors declare that the research was conducted in the absence of any commercial or financial relationships that could be construed as a potential conflict of interest.

Received: 11 January 2010; paper pending published: 10 March 2010; accepted: 07 April 2010; published online: 14 May 2010

Citation: QiXL, Katsuki F, Meyer T, Rawley JB, Zhou X, Douglas KL and Constantinidis $C$ (2010) Comparison of neural activity related to working memory in primate dorsolateral prefrontal and posterior parietal cortex. Front. Syst. Neurosci. 4:12. doi: 10.3389/fnsys.2010.00012

Copyright (C) 2010 Qi, Katsuki, Meyer, Rawley, Zhou, Douglas and Constantinidis. This is an open-access article subject to an exclusive license agreement between the authors and the Frontiers Research Foundation, which permits unrestricted use, distribution, and reproduction in any medium, provided the original authors and source are credited. 